\title{
The Asilomar Conference on Ion Spectroscopy, October 16-20, 2009
}

I t could easily be assumed that researchers in the field of ion spectroscopy would be a homogeneous group whose members are fully aware of developments in this specialized field. However, the recently concluded Asilomar Conference on Ion Spectroscopy has proven this wrong. In fact, the diversity of research directions in ion spectroscopy is quite broad. Thus the convergence of this disparate group of spectroscopists and theorists at Asilomar resulted in a stimulating and exciting interchange of information and ideas, which fully met the goals of these workshops. It is notable that much work in this field is being done by European scientists (many of whom are active in ASMS activities), and the conference was enriched by a sizable contingent of European speakers and participants.

Several spectroscopic themes were represented. The largest group of presenters described diverse experiments in which ions are generated in a suitable ion source, and their spectroscopy is probed using a variable wavelength light source and/or detector.

A number of the participants were users of two infrared free electron laser (FEL) facilities, CLIO in Orsay, France, and FELIX at the FOM in Utrecht, Netherlands. Most of these experiments utilized electrospray ionization to generate biologically interesting ions, and were directed at elucidating the structure of ions by comparing the predicted IR spectra from calculated structures with the experimentally observed spectra. The high power of the IR FEL permits multiphoton absorption so that ions are dissociated. The absorption is thus monitored by collecting dissociation products as the laser is scanned. These two FEL facilities are notable for the breadth of chemistry brought by their numerous outside users. Among the chemistry topics surveyed in conference talks were uranyl complexes (Gary Groenewold, Idaho National Laboratory), carbon clusters and metal/metal oxide clusters (Gerard Meijer, Fritz-Haber Institut), cationized nucleobases (Mary Rodgers, Wayne State University), anionic species (Jos Oomens, FOM/FELIX), and inorganic and organic complexes (Philippe Maitre, CLIO).

Closely connected with the experimental infrared studies of moderate-sized gas-phase ions are the parallel computational explorations of their possible conformations, thermochemistry, and IR spectroscopy. Two frequent FEL collaborators who are advancing this computational endeavor are Gilles Ohanessian (Ecole Polytechnique) and Bela Paizs (German Cancer Research Center), who discussed the advancing methodology and results of conformational searches, with a focus on peptide systems.
Both CLIO and FELIX store the ions at room temperature in ICR traps. These experiments are contrasted with laboratory based experiments, as described by Thomas Rizzo (EPFL Lausanne) in which ions are stored in an ion trap that has been cooled to about $12 \mathrm{~K}$ by a He cryostat, and the ions are interrogated by a tunable IR OPO laser. Ions are cooled to this low temperature by cold helium collisions and by allowing them to establish radiative equilibrium with the walls of the trap. The IR spectroscopy of the ions is again monitored by multiphoton dissociation, which may in some experiments be assisted by a fixed-frequency $\mathrm{CO}_{2}$ laser, or more gently by absorption of a UV photon in a two photon experiment. These high-resolution IR experiments are uncovering and spectroscopically resolving multiple low-temperature conformers of biomolecule ions. The other approach for cooling the ions involves the use of pulsed molecular beams, in which ions are created by a discharge close to the nozzle exit and the soup of ions is subsequently cooled in the expansion. Ions are then mass selected and interrogated by an IR OPO laser using Ar tagging to monitor the photon absorption step. In this method, Ar is weakly bound to the cold ion and thus readily departs upon the absorption of one IR photon. Michael Duncan (University of Georgia) described examples of both metal-ion complexes and also small hydrocarbon ions exploiting this powerful spectroscopic approach. Evan Bieske (University of Melbourne) described results using this approach for metal-ion hydrogen complexes, envisioning applications (somewhat in the future) to hydrogenstorage technology. In both trapped-ion work and molecular beam work, the combination of cooled ions and the high resolution of the OPO lasers $\left(0.1-0.01 \mathrm{~cm}^{-1}\right)$ results in IR spectra that are much better resolved than is possible with the IR FEL with its resolution of $10 \mathrm{~cm}^{-1}$.

Progress was reported from two labs whose spectroscopy encompasses both the vibrational transitions in the infrared, and the electronic transitions in the visible/UV. Ricardo Metz (University of Massachusetts) discussed recent extensions of his visible/UV work with catalytically interesting transition-metal complexes, involving infrared light. And John Maier (University of Basel) surveyed the wide-ranging activities of his lab using a remarkable array of ion-spectroscopic approaches, with the theme of learning more about astrochemically interesting ions.

The transition from gas-phase ion chemistry to ions in solution is increasingly accessible to experiment. Two groups pioneering the study of these connections gave 


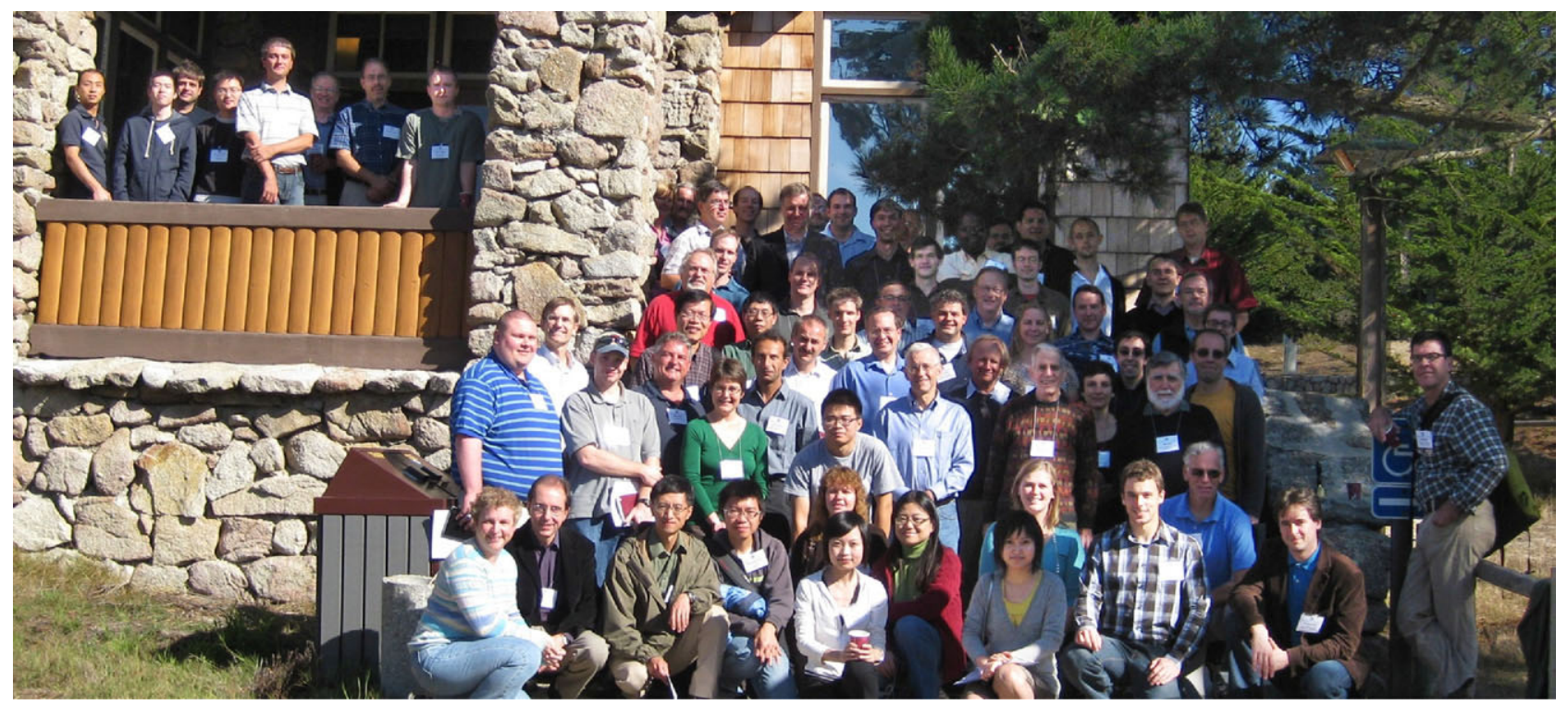

updates of their progress. Mark Johnson (Yale University) described some of their recent work on water clusters, and Evan Williams (University of California, Berkeley) discussed their "nanocalorimetry" experiments on water-clustered metal ions creating novel links between gas-phase ion thermochemistry and solutionphase electrochemistry. Dissociation reactions induced by black-body thermal radiation, external light sources, and electron capture are quantified as the clusters approach the large-size limit, where their thermodynamics can be smoothly joined to the thermodynamics of bulk aqueous solution.

A second category of ion spectroscopy starts from neutral precursors, and measures the energy spectrum of the emitted photoelectrons. This approach requires a high energy photon to ionize the sample. The traditional low resolution He I PES has been supplanted by threshold ionization methods such as pulsed field ionization or threshold photoionization with velocity focusing optics, which result in electron energy resolutions that are limited only by the photon source bandwidth, which in the case of pulsed lasers is sub $\mathrm{meV}$. These studies are carried out at an increasing number of synchrotron radiation facilities, represented at this workshop by researchers from Soleil in Paris, and the Swiss Light Source in Villigen, Switzerland. The PFI and related MATI experiments utilize the usual array of lasers that are housed in individual research laboratories. One major difference between these far-UV photoelectron experiments, and the IR and visible laser optical spectroscopy experiments summarized above, is in the nature of the molecules accessible to study. Only open shell ions that have the structure of the neutral precursors can be investigated in the photoionization studies. On the other hand, ions studied in the ion trap and molecular beam techniques can be generated by a wide variety of ion sources, including electrospray and by discharge in a supersonic expansion. These latter ions can be closed shell ions, ionic clusters, and reaction product and fragment ions, giving rise to the diversity of chemical systems surveyed in the presentations at the conference.

Several of the presenters of photoelectron spectroscopy studies are involved with the large user-friendly synchrotron light sources in Europe. Ivan Powis (University of Nottingham) and Laurent Nahon (SOLEIL, Saint-Aubin) both described vibrational circular dichroism studies made possible by the polarized-beam capabilities of the synchrotron. Andras Boedi (Paul Scherrer Institut) described electron/ion coincidence experiments at the Swiss Light Source in which mass selected threshold photoelectron spectra with $2 \mathrm{meV}$ resolution were obtained from a mixture of molecules in the molecular beam. Laboratory laser studies were presented by Katharine Reid (University of Nottingham) who showed state-of-the-art velocity imaging capabilities used to determine complex angular distributions in multi-photon ionization studies of small molecules. Cheuk-Yiu Ng (University of California, Davis) described advanced experimental approaches involving vacuum ultraviolet/IR laser combinations, which yielded rotationally resolved spectra from which ion structures could be established. Finally, Dong-Sheng Yang (University of Kentucky) discussed ZEKE experiments, using one photon ionization of organometallic complexes.

A final group of researchers presented groundbreaking results on light emission from ions, encompassing fluorescence, using tagging of biomolecules by a fluorophore (Joel Parks, Rowland Institute at Harvard), FRET, and photodissociation probing of trapped chromophoric ions (Rebecca Jockusch, University of Toronto), and surface enhanced Raman studies (Frank 
Turecek, University of Washington) of ions deposited on a surface.

Peripheral high points of the presentations were the Biblical reference to the adventure of Pharoah in the Red Sea (Williams), and an excursion into stereochemical considerations in mating activities of optically asymmetric snails (Powis).

Six presenters in the "Hot Topics" sessions gave short presentations of their recent work. Jeffrey Steill (FOM), Mathias Schaefer (Cologne), James Prell (Berkeley), Nick Polfer (Florida) and John Eyler (Florida) discussed IRMPD results for a variety of ions, and Jana Roithova (Charles University) described ion-molecule chemistry of hydrocarbon dications.

The strong ties between ion spectroscopy and astrochemistry were brought out in John Maier's presentation noted above, but most comprehensively in the broad-ranging and very popular after-banquet talk by Eric Herbst (Ohio State University).
This Special Focus on Ion Spectroscopy by the Journal of the American Society for Mass Spectrometry reflects the diversity and excitement of the presentations at the 2009 Asilomar Conference.

Tomas Baer Co-organizer, 2009 Asilomar Conference on Ion Spectroscopy University of North Carolina Chapel Hill, North Carolina

Robert C. Dunbar Co-organizer, 2009 Asilomar Conference on Ion Spectroscopy

Case Western Reserve University Cleveland, Ohio

Veronica M. Bierbaum Associate Editor, JASMS University of Colorado Boulder, Colorado 